\title{
Regression of left ventricular hypertrophy in a transplanted heart
}

\author{
S C D Grant, A N Rahman, N H Brooks
}

\begin{abstract}
A woman with advanced coronary artery disease underwent heart transplantation. The donor heart had left ventricular hypertrophy. The electrocardiographic and echocardiographic evidence of left ventricular hypertrophy regressed during follow up; estimated

left ventricular mass decreased from $393 \mathrm{~g}$ to $171 \mathrm{~g}$. The adaptation of myocardial mass and performance after transplantation is not fully understood. This case illustrates the potential for regression of left ventricular hypertrophy in response to altered loading conditions.
\end{abstract}

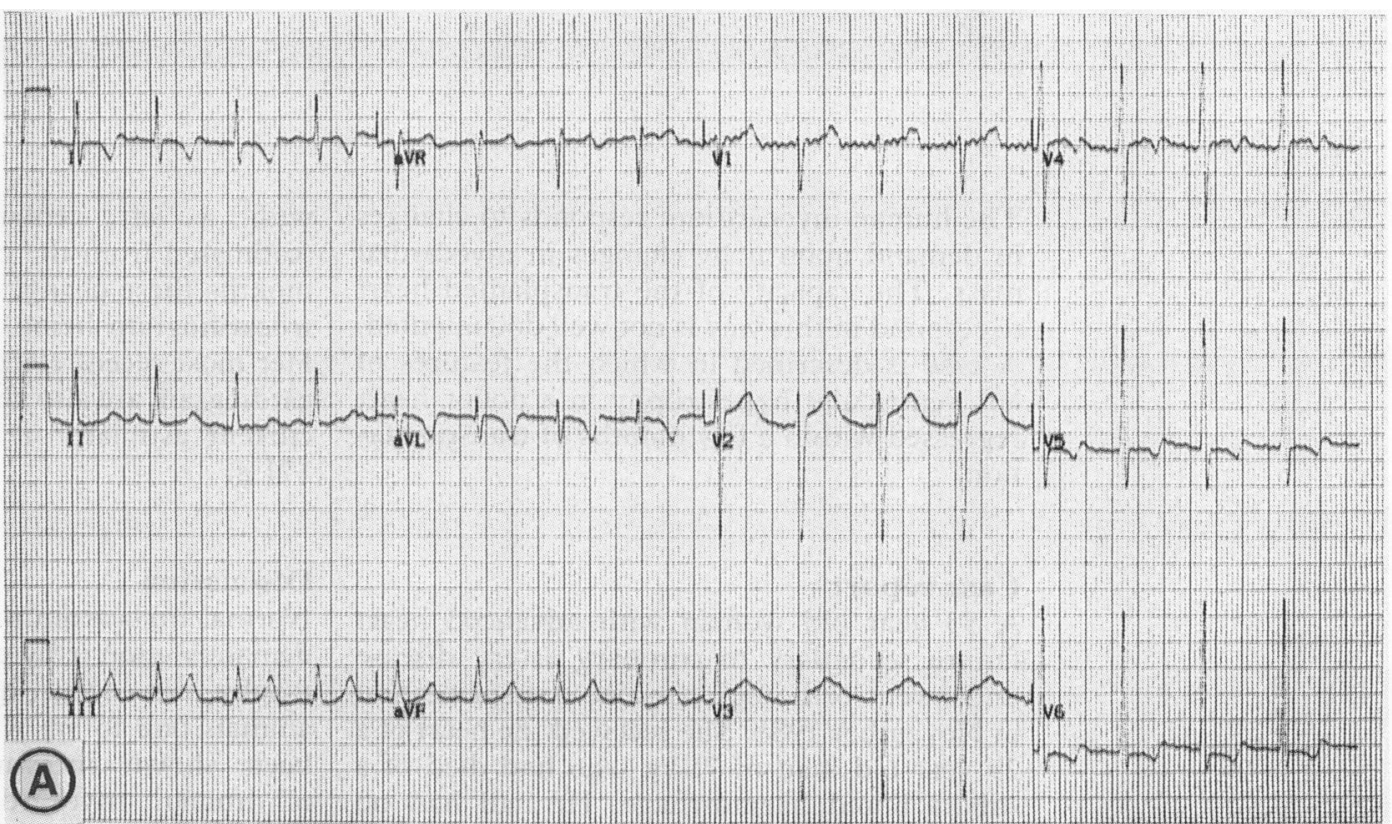

Wythenshawe Hospital, Manchester S C D Grant A N Rahman N H Brooks

Correspondence to Dr S C D Grant,

Department of Cardiology, Wythenshawe Hospital, Wythenshawe Hospital,
Manchester M23 9LT.

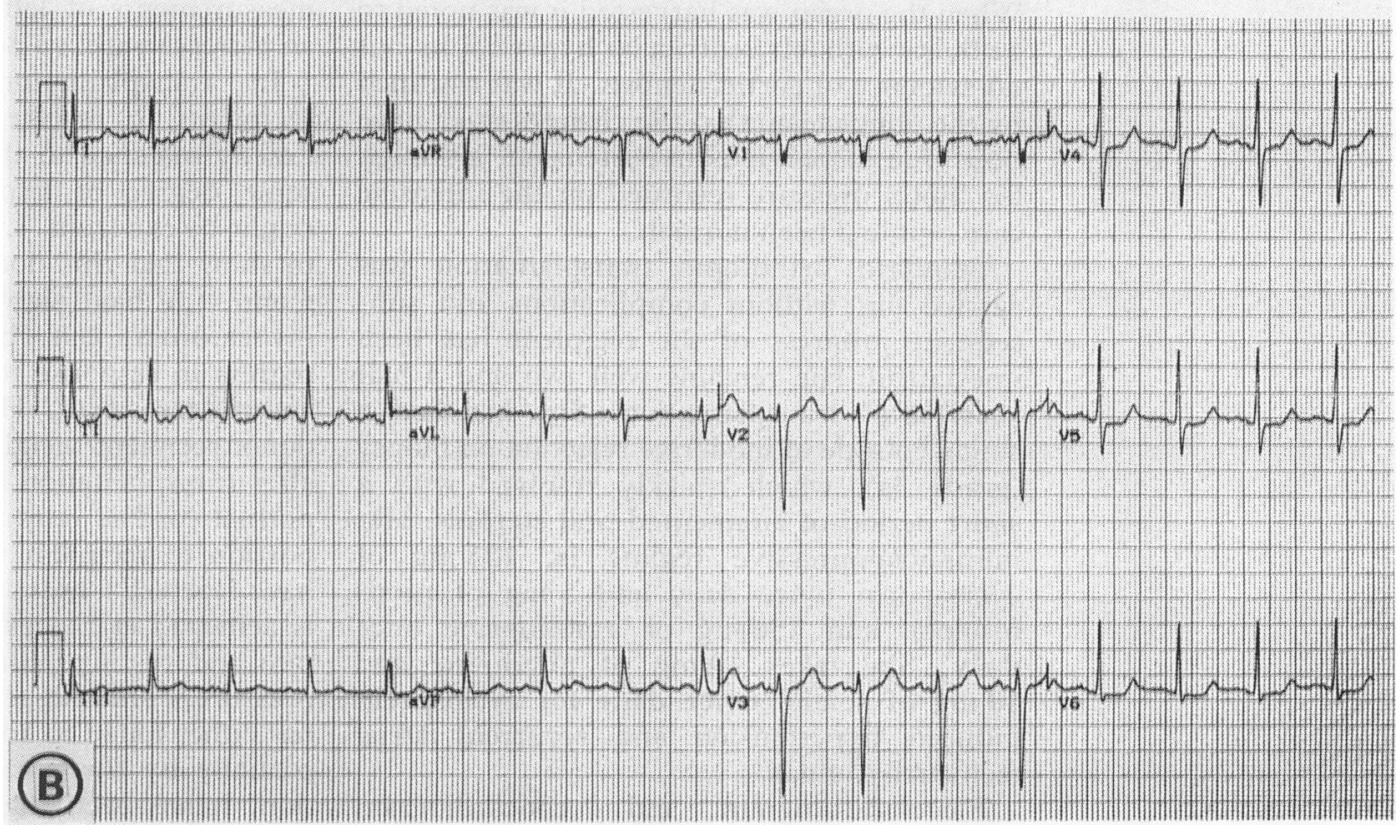

Figure 1.12 lead electrocardiograms: ( A) Immediately after transplantation, (B) 25 months after transplantation. Comparison shows a reduction in left ventricular hypertrophy. 
Figure 2 M Mode echocardiograms at the level of the left ventricle: (A) Immediately after transplantation, $(\boldsymbol{B}) 25$ months after

transplantation.

Comparison shows a

considerable reduction in

left ventricular posterior

wall and septal thickness.

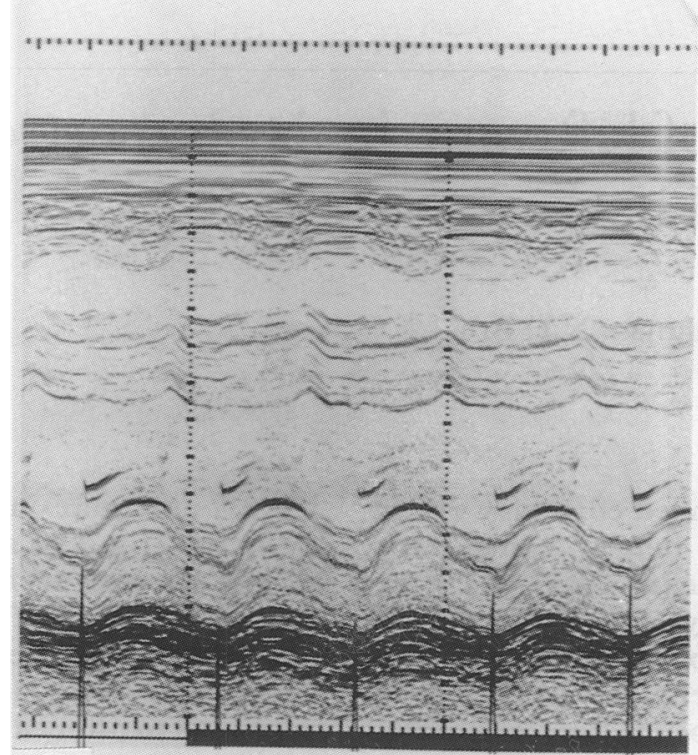

(A)

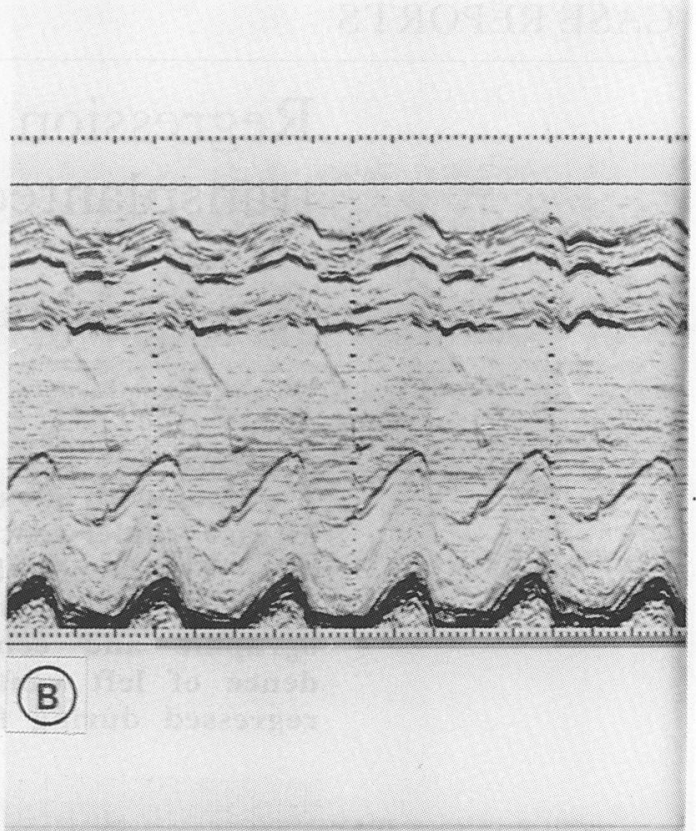

with a left ventricular mass of $393 \mathrm{~g}$ (estimated from the cube rule ${ }^{1}$ ). Over several months these changes resolved with the electrocardiogram being normal at nine months. Her most recent electrocardiogram and echocardiogram are also shown in figs 1 and 2 . The current estimate of left ventricular mass is $171 \mathrm{~g}$.

\section{Discussion}

\section{Case report}

A 53 year old woman with advanced heart failure secondary to coronary artery disease underwent orthotopic cardiac transplantation in November 1988. She received the heart of a 29 year old man of $71 \mathrm{~kg}$ who had died of a subarachnoid haemorrhage. He had had no medical history. His chest $x$ ray was normal. When the organ was harvested it was noted to be bulky with left ventricular hypertrophy but no evidence of aortic stenosis. It was assumed by the donor team that the changes must have been due to previous hypertension, although his blood pressure recorded on the intensive care unit had been normal.

Standard orthotopic transplantation was carried out without complications and subsequent progress of the recipient has been uneventful; she remains well two and a half years after transplantation. She has had a single episode of moderate rejection on biopsy (grade 3a), which quickly resolved with a short period of augmented oral steroids. Her immunosuppressive regime is; $125 \mathrm{mg}$ of cyclosporin twice daily and $5 \mathrm{mg}$ of prednisolone and $150 \mathrm{mg}$ of azathioprine once daily. She takes $10 \mathrm{mg}$ of nifedipine and $40 \mathrm{mg}$ of sotalol twice daily for cyclosporin induced hypertension and recurrent atrial tachyarrhythmias.

Electrocardiogram and echocardiogram immediately after the transplantation (figs 1 and 2) showed left ventricular hypertrophy
Normal myocardium responds to changes in trophy can occur quickly in experimental animals one to six weeks ${ }^{23}$ and this has also been shown in studies on the response of human myocardium to training ${ }^{4}$ and pregnancy, ${ }^{5}$ when again echocardiographic measurements of left ventricular mass show an increase within a period of weeks.

In transplantation it is unlikely for recipients to receive a donor heart that is exactly matched to their needs and therefore some degree of hypertrophy or involution might be expected. Little work has been done to show that this does in fact occur. Angermann et al have shown that the transplanted myocardium can both hypertrophy and regress in response to recipient hypertension and it's treatment. ${ }^{6}$ Hosenpud et al compared donor:recipient weight ratio at three months after transplantation and found that although normal cardiac output is maintained in proportion to recipient weight, in patients who have received a heart from a lighter donor this is achieved at the expense of a higher heart rate and resting pulmonary capillary wedge pressure. ${ }^{7}$ This suggests that the myocardium has either failed to adapt, or has done so only partially by three months after transplantation. It has been suggested that this relation does not persist at 12 months, ${ }^{8}$ which would be in keeping with the idea of gradual adaptademands upon it by changes in mass. Hyper- 
tion of the donor heart to its new loading conditions over a period of three to 12 months. A recent report retrospectively assessed the effect of donor:recipient weight mismatch on outcome and suggested that, contrary to popular belief, oversizing (that is a donor weight in excess of recipient ideal weight) results in a poorer outcome. ${ }^{8}$ There was a continuous (negative) relation between degree of oversizing and survival. It was also concluded that undersizing was not detrimental to allograft function and survival.

Further work on the correlation between size match and ultimate outcome is required to define the optimal limits of size matching.

1 Monaghan M. Practical echocardiography and Doppler. Chichester: John Wiley, 1990.
2 Sasayama S, Ross J Jr, Franklin D, Bloor CM, Bishop S, Dilley RB. Adaptations of the left ventricle to chronic pressure overload. Circ Res 1976;38:172-8.

3 McCullagh WH, Covell JW, Ross J Jr. Left ventricular dilatation and diastolic compliance changes during chronic volume overloading. Circulation 1972;45:943-51.

4 DeMaria AN, Neumann A, Lee G, Fowler W, Mason DT Alterations in ventricular mass and performance induced Alterations in ventricular mass and performance induced graphy. Circulation 1978;57:237-44.

5 Katz R, Karliner JS, Resnik R. Effects of a natural volume overload state (pregnancy) on left ventricular performance in normal human subjects. Circulation 1978;58: 434-41.

6 Angermann CE, Spes C, Hart RJ, Kemkes BM, Theisen $\mathrm{K}$. Effects of enalapril, furosemide and verapamil in hypertension after orthotopic cardiac transplantation. [Abstract]. J Heart Transplant 1988;7:77.

7 Hosenpud JD, Pantely GA, Morton MJ, Norman DJ, Cobanoglu AM, Starr A. Relation between recipient: donor body size match and hemodynamics three months after heart transplantation. J Heart Transplant 1989; 8:241-3.

8 Costanzo-Nordin MR, Liao Y, Grusk BB, et al. Oversizing of donor hearts: Beneficial or detrimental? J Heart Transplant 1991;10:717-30. 